\title{
HAND-ASSISTED LAPAROSCOPIC NEPHRECTOMY AS A MINIMALLY INVASIVE OPTION IN THE TREATMENT OF LARGE RENAL SPECIMENS
}

\author{
M. TOBIAS-MACHADO, ALESSANDRO TAVARES, PEDRO H. FORSETO JR, JOAO P. \\ ZAMBON, ROBERTO V. JULIANO, ERIC R. WROCLAWSKI
}

Section of Urology, ABC School of Medicine, Santo Andre, Sao Paulo, Brazil

\begin{abstract}
Introduction: We describe our experience with hand-assisted laparoscopy (HAL) as an option for the treatment of large renal specimens.

Materials and Methods: Between March 2000 and August 2004, 13 patients candidate to nephrectomies due to benign renal conditions with kidneys larger than $20 \mathrm{~cm}$ were included in a prospective protocol. Unilateral nephrectomy was performed in cases of hydronephrosis (6 patients) or giant pyonephrosis (4 patients). Bilateral nephrectomy was performed in 3 patients with adult polycystic kidney disease (APKD) with low back pain refractory to clinical treatment previous to kidney transplant. The technique included the introduction of 2 to $310 \mathrm{~mm}$ ports, manual incision to allow enough space for the surgeon's wrist without a commercial device to keep the pneumoperitoneum. The kidney was empty, preferably extracorporeally, enough to be removed through manual incision. We have assessed operative times, transfusions, complications, conversions, hospital stay and convalescence.

Results: The patients mean age ( 9 women and 4 men) was 58 years. Mean operating time was $120 \pm 10 \mathrm{~min}$ (hydronephrosis), $160 \pm 28 \mathrm{~min}$ (pyonephrosis) and $190 \pm 13 \mathrm{~min}$ (bilateral surgery for APKD). There was a need for a conversion in 1 case and another patient needed a transfusion due to a lesion in the renal vein; 2 patients had minor complications.

Conclusions: HAL surgery is a minimally invasive alternative in the treatment of large renal specimens, with or without significant inflammation.
\end{abstract}

Key words: nephrectomy; laparoscopy; pyelonephritis; hydronephrosis; polycystic kidney, autosomal dominant

Int Braz J Urol. 2005; 31: 526-33

\section{INTRODUCTION}

Some authors have considered the presence of large renal specimens or inflammatory kidney disease as contraindications regarding the use of laparoscopy. The difficult access to the kidney limits, the contiguous fibrosis and the difficult in identifying the renal vessels lead to longer operating times and higher complication and conversion rates when surgery is performed through pure laparoscopic technique $(1,2)$.

In 1997, Nakada et al. performed the first hand-assisted laparoscopic nephrectomy (HAL) in humans (3). Since then, even if not in a consensual manner, this access has become an alternative to more difficult nephrectomies. 
Some studies in nephrectomy for kidney donation have demonstrated that HAL can be superior to the open technique and similar to the exclusive laparoscopic one when we take into consideration postoperative recovery $(4,5)$. In the case of kidneys with severe inflammation, there must be a significant reduction in operating times, with minimum differences in terms of morbidity when we compare HAL with the exclusive laparoscopic technique (6).

The aim of the present study was to report the experience in the treatment of 16 giant renal specimens utilizing HAL, discussing the advantages of this procedure and comparing the results with literature results.

\section{MATERIAL AND METHODS}

\section{Selection Criteria and Sample Descriptions}

In the period from March 2000 to August 2004, 13 patients with giant kidneys (more than 20 $\mathrm{cm}$ in the largest diameter or crossing the midline) candidates of nephrectomy due to benign renal conditions were submitted to HAL nephrectomy and followed through a prospective protocol. A computerized tomography of the abdomen was systematically performed, assessing kidney size, hilum position (frequently altered due to the distortion because of the exaggerated volume of these kidneys), and the degree of inflammation, through the perirenal fat smear.

Indications for unilateral nephrectomy were pyonephrosis in 6 patients (one of them with adult polycystic kidney disease - APKD) and giant hydronephrosis without a significant inflammatory component (nonfunctioning kidneys with stenosis of the ureteropelvic junction) in 4 patients. Bilateral nephrectomy was performed in 3 patients bearers of terminal renal insufficiency due to APKD previously to the kidney transplant, with low back pain refractory to clinical treatment. All patients with renal insufficiency were submitted to dialysis the day before the operation.

No colon preparation was utilized, being employed $1 \mathrm{~g}$ of cephalotine as an antibiotics prophylaxis in the anesthetic induction, repeated every 6 hours in the first $48 \mathrm{~h}$.

\section{Operative Technique}

Two surgeons assisted by resident physicians in training operated all patients included. For the unilateral nephrectomy, the patient was placed in a lateral decubitus position with the kidney to be operated facing upwards. An internal pararectal incision of approximately $6 \mathrm{~cm}$ exact for the surgeon's wrist and 2 laparoscopic ports were used (Figure-1). We

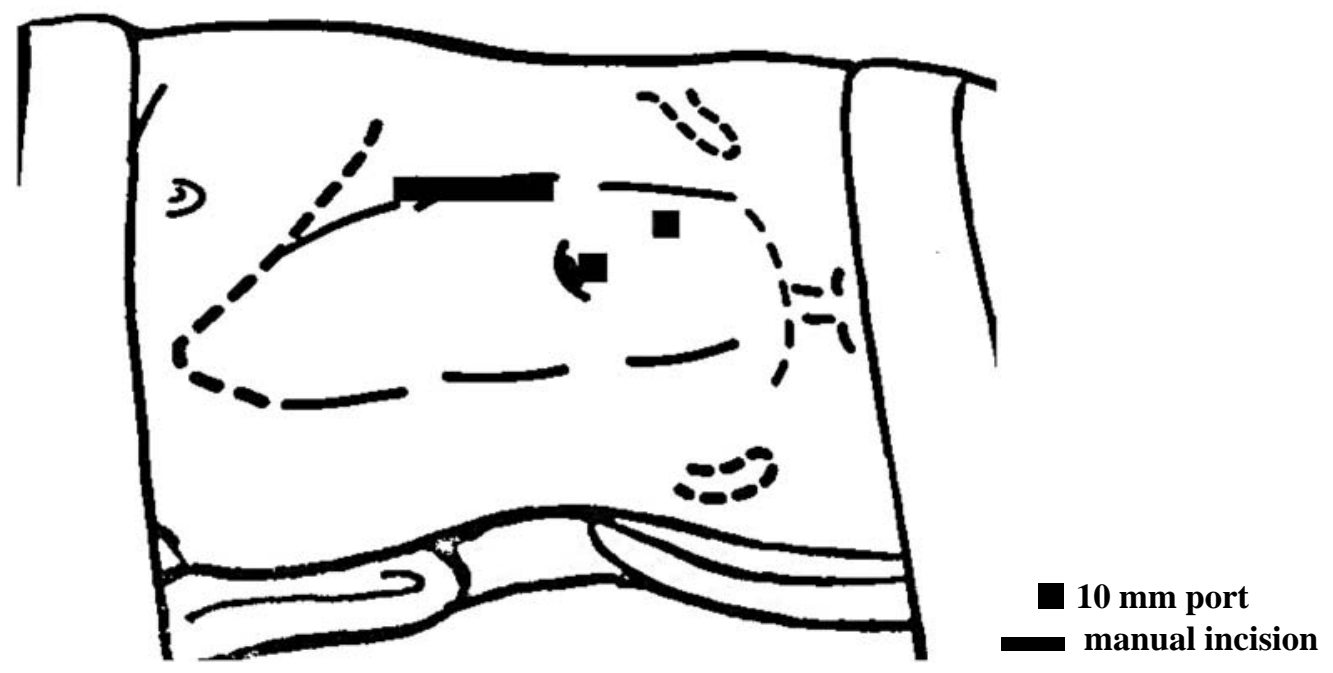

Figure 1 - Unilateral nephrectomy: position of the laparoscopic ports and the manual incision. 


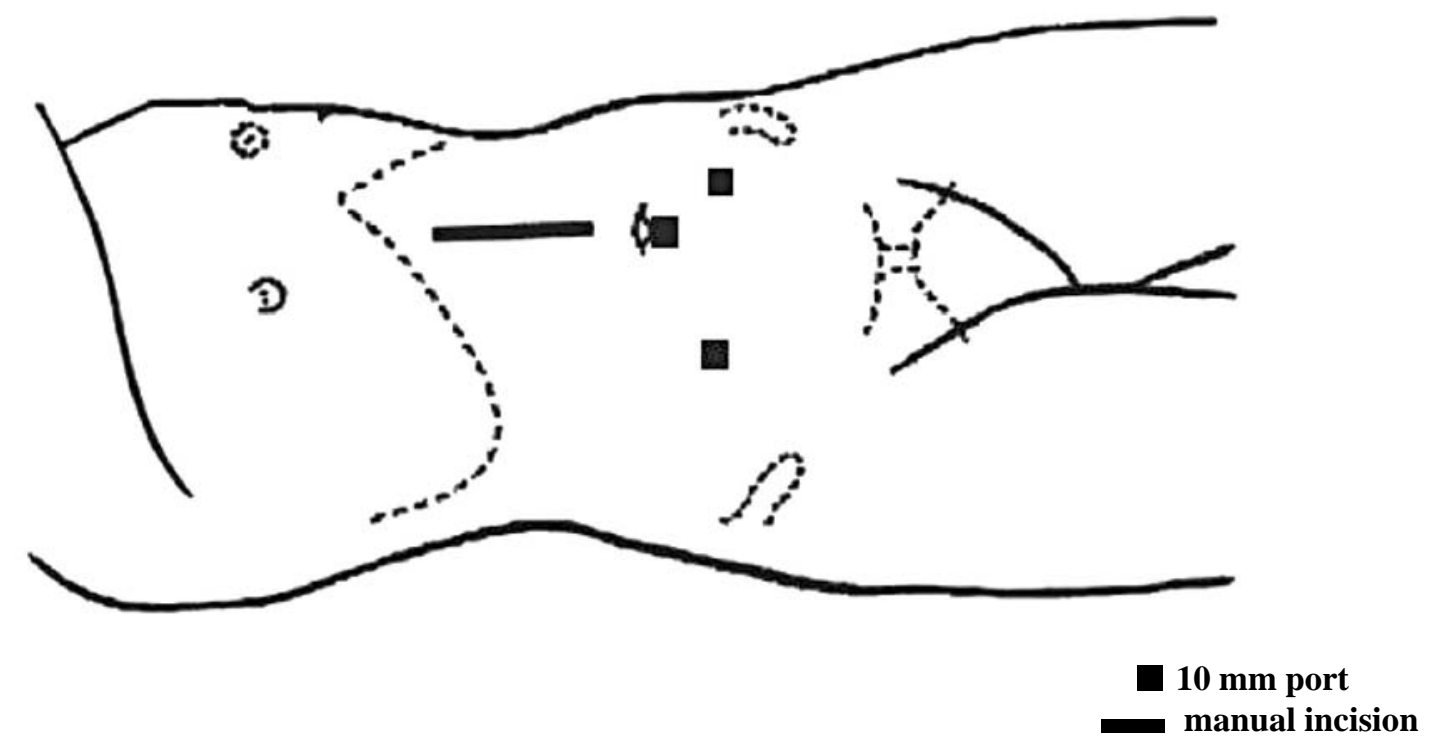

Figure 2 - Bilateral nephrectomy: position of the laparoscopic ports and the manual incision.

have planned the incision over the projection of the renal vessels with the help of a preoperative tomography.

In the cases of bilateral nephrectomy, the patient was placed in a horizontal dorsal decubitus position, and he/she was fixed to the table in order to enable its lateral mobilization according to the side to be approached. One sole incision in the midline was utilized to both side nephrectomies. Three $10 \mathrm{~mm}$ ports were utilized (Figure-2).

To reduce the costs, permanent laparoscopic material was employed and no commercial device to keep the pneumoperitoneum was used. The renal pedicle was controlled by a Hem-o-lok ${ }^{\circledR}$ polymer clip (Weck Closure Systems, Research Triangle Park, NC, USA) proximal and distal both for the artery and for the vein. In the impossibility of laparoscopic control of the pedicle, we have placed long Doyen valves through the manual incision and have performed the individualized extracorporeal ligature of the vessels. When the presence of inflammation hindered the individualization of the vessels, we have completely mobilized the kidney through a manual dissection for a further application of Satinsky clamp, pedicle sec- tion and suture with polypropylene through the previously planned incision. The removal of the specimen was done through the hand incision (Figure-3), without previously placing it in a sac, after an extracorporeal drainage (hydronephrosis without infected content) or multiple extracorporeal punches (pyonephrosis or APKD). The manual incision and the ports aponeurosis were either closed with polypro-

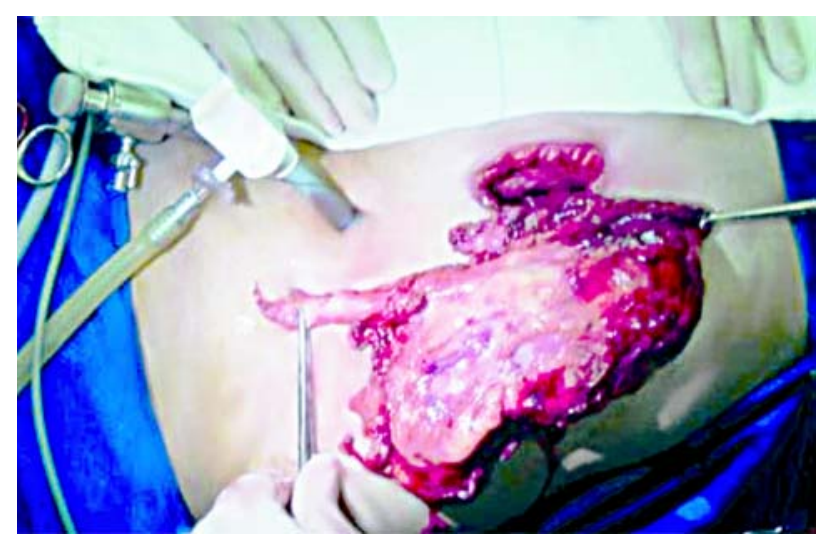

Figure 3 - Surgical specimen (pyonephrosis) removed through the manual incision. 
pylene 0 and the skin with nylon 4-0 (when the possibility of infection was considered) or with intradermal suture with monocryl 5-0. In the presence of pyonephrosis, the manual incision was systematically washed with saline and a Penrose drain was placed through a $10 \mathrm{~mm}$ port.

Patients were followed-up at an outpatient clinic by the surgeon on days 7, 30, 60 and 90 postoperatively.

\section{RESULTS}

The main results obtained in this group of patients can be verified in Table- 1 .

Due to the reduction of working space, there is a constant need for the surgeon to orient the assistant for a correct optical direction to the field that we intend to approach.

We have observed that the pneumoperitoneum can be maintained in all cases. The mean number of times that the hand had to be taken out the cavity was 2 ( 1 to 4 ) times per kidney, mainly for the exchange of compresses or for the performance of external maneuvers. Since there was no mechanism to contain the gas, there was a need to wait until insufflation was renewed to restart surgery. This happened without any harm to the procedure. The compression of the surgeon's wrist through the aponeurosis was not troublesome when the surgery for the kidney occurred in up to 2 hours. The control of the renal pedicle was obtained through exclusive laparoscopic maneuvers in 11 kidneys. In 3 kidneys (pyonephrosis), the application of a Satinsky clamp was needed after the whole kidney was mobilized. In a patient with APKD, we have chosen the ligature and section of the vessels of the 2 kidneys after external and bilateral individualization through the manual incision. In these cases, an additional enlargement of the incision was needed for the control of the renal pedicle.

Operative times were $120 \pm 10$ min (hydronephrosis), $160 \pm 28 \mathrm{~min}$ (pyonephrosis) and $190 \pm$ 13 min (APKD).

No case of simple hydronephrosis presented complications. Two cases presented major complications (vascular lesion, one with transfusion and another followed by conversion) and 2 cases minor complications (asymptomatic pneumothorax and wound infection, followed by hernia).

A patient with pyonephrosis presented a lesion in the right renal vein during the manual displacement of the kidney. The lesion was tamponade with the help of a hand and controlled with a polymer clip. Patient received intraoperatively 2 red blood cell concentrates without the need for conversion, being discharged from the hospital in the third day postoperatively.

One of the APKD patients developed a right pneumothorax, drained in the immediate postoperative. The drain was taken out after 48 hours and the patient was dismissed in the 4th postoperative day without any complication.

Table 1 - Data obtained from 13 patients submitted to hand-assisted laparoscopic nephrectomy.

\begin{tabular}{|c|c|c|c|c|c|c|c|}
\hline Pathology & $\begin{array}{c}\mathrm{N} \text { of } \\
\text { Kidneys }\end{array}$ & $\begin{array}{l}\text { Mean Kidney } \\
\text { Length }\end{array}$ & $\begin{array}{l}\text { Operative } \\
\text { Time }\end{array}$ & $\begin{array}{c}\text { Major } \\
\text { Complications }\end{array}$ & $\begin{array}{c}\text { Minor } \\
\text { Complications }\end{array}$ & $\begin{array}{c}\text { Conversion } \\
\text { Rate }\end{array}$ & $\begin{array}{c}\text { Convalescence } \\
\text { Time }\end{array}$ \\
\hline \multicolumn{8}{|l|}{ Giant } \\
\hline hydronephrosis & 4 & $21 \mathrm{~cm}$ & $120 \min *$ & 0 & 0 & 0 & 25 days \\
\hline \multicolumn{8}{|l|}{ Giant } \\
\hline pyonephrosis & 6 & $21 \mathrm{~cm}$ & $160 \min *$ & $33 \%$ & $16.6 \%$ & $16.6 \%$ & 31.7 days \\
\hline APKD & 6 & $32 \mathrm{~cm}$ & $190 \min * *$ & 0 & $16.6 \%$ & 0 & 25 days \\
\hline Total & 16 & $25.12 \mathrm{~cm}$ & & $12.5 \%$ & $12.5 \%$ & $6.2 \%$ & 25 days \\
\hline
\end{tabular}

* operative times corresponding to unilateral nephrectomy; ** operative times corresponding to bilateral nephrectomies; APKD = adult polycystic kidney disease. 
One of the patients with morbid obesity and pyonephrosis developed a surgical wound infection, satisfactorily treated with local care. In the late follow-up, he presented an incisional hernia surgically corrected with a Marlex mesh.

In one of the cases, a conversion to open surgery was necessary. This 72 year-old patient presented APKD and a clinical picture of a recurrent right pyelonephritis. During the parietocolic gutter displacement, we have observed an intense adhesion to the right colon, impeding access to the renal pedicle in the initial operative time. The dimension of this kidney that overcame the midline, promoted an additional difficulty to the endoscopic procedure, ending up in a tactical conversion through an enlargement of the manual incision, from the costal edge to the right iliac fossa. Total operative time was $192 \mathrm{~min}$, being discharged in the 7th postoperative day. The return to normal activities occurred 65 days after surgery.

The other patients were discharged between the 1 st and the 5 th postoperative day (mean 3.4 days). Thirty days after the surgery (mean 25 days), all patients, exception made to the case that needed conversion, were totally recovered to normal activities.

\section{COMMENTS}

Laparoscopic renal surgery is the gold standard treatment for renal ablation of benign non-inflammatory pathology. Even though, in some cases of large renal specimens or associated to intense inflammatory process, renal laparoscopic surgery still presents some restrictions. Although this is a subject of great controversy in literature, some authors report that in these situations, pure laparoscopic surgery presents operative time, complication and conversion rates superior to the habitual ones, being the benefits in these cases not as notorious. On the other hand, the conventional technique in these conditions frequently requires either a lumbar or a median incision of large dimensions.

HAL surgery appears as an attractive alternative in these cases, adding up the advantages of a minimally invasive treatment to the possibility of a faster and safer surgery. It allows the introduction of the surgeon's hand in the operative field, making it easier maneuvers of dissection, retraction and hemostasia while keeping both tactile and spatial sensations. In case of a vascular accident, the control can be easily obtained through a digital compression of the vessel, allowing, if necessary, a conversion in non-emergency conditions. Besides, one or more compresses can be introduced in the cavity to tamponade a certain region while dissection continues $(7,8)$.

We believe that hand-assisted technique has a precise indication in cases where technical difficulty is previewed. In kidneys with an accentuated inflammation, Wolf et al. have demonstrated a significant reduction in operative time comparing HAL with conventional laparoscopy, with minimum differences in terms of morbidity (6).

Although there are evident advantages as to the comfort during the procedure, the cost of devices to keep the pneumoperitoneum during HAL as the Hand Port System ${ }^{\circledR}$ (Smith and Nephew, Inc., Andover, MA, USA), the Intromit hand assistance device ${ }^{\circledR}$ (Applied Medical, Santa Margarita, CA, USA), the Lapdisc ${ }^{\circledR}$ (Ethicon Inc., Cincinnati, OH, USA) or the Pneumo Sleeve ${ }^{\circledR}$ (Dexterity, Roswell, GA, USA), it can be a limiting factor in most of public services in developing countries. Due to institutional questions, we utilized HAL without the assistance of special devices. We could notice that this procedure is feasible though an incision large enough to fit the surgeon's wrist, with the maintenance of the pneumoperitoneum, which is lost only momentarily when there is a need for a large mobilization, the retrieval or exchange of the surgeon's hand.

Regarding the surgical indication, it was demonstrated in this series that the size of the kidney in the absence of inflammation is not a factor of contraindication to the laparoscopic procedure. Giant hydronephrosis has been defined as a kidney that has more than $1000 \mathrm{~mL}$ of fluid in its collecting system (9). This group of patients was submitted to the procedure with a very satisfactory operative time. In this specific indication, pure laparoscopy can also be performed safely, however with a longer operative time. Morbidly obese, where pure laparoscopic surgery can present higher technical difficulty, and patients with precarious clinical conditions, where a short opera- 
tive time added to a minimally invasive surgery is essential, represent 2 sub-groups where access to HAL can have an additional advantage over the exclusive laparoscopic access.

Laparoscopic nephrectomy in cases of pyonephrosis is a highly complex surgery. The difficult visualization of the dissection plans, the inflammation of the tissue, the adherence of vital structures (colon, duodenum, liver, gallbladder and great vessels) and the obliteration of the renal hilum are factors that make this procedure challenging. In all pyonephrosis cases in this study, kidneys presented more than $20 \mathrm{~cm}$ in the biggest axis and signs of perirenal fat infiltration in the preoperative computerized tomography. For all these aggravating factors most part of the procedures can be performed without the need for conversion to the open procedure. Digital renal dissection in the subcapsular plane and, eventually, the extracorporeal ligature of the pedicle with a Satinsky clamp, in face of great difficulties, are alternatives that can avoid the conversion to the conventional technique.

APKD is a common genetic disorder that is inherited as an autosomal dominant disease, characterized by multiple bilateral renal cysts, nonfunctioning and noncommunicating. Nephrectomy in APKD can be necessary when the native kidney occupies all the iliac fossa where the kidney will be transplanted, or due to hypertension or refractory pain, hematuria requiring transfusion and recurrent infections, symptoms that appear between the third and fourth decades of life. Around 50\% of the patients develop terminal renal insufficiency up to 60 years old, needing dialysis or renal transplant procedures (10). The rate of complications associated to conventional open procedure is significant, with 12 $\%$ morbidity and $5 \%$ mortality rate (11). These numbers were responsible for a decrease in nephrectomy for APKD patients between 1970 and 1980 (12,13).

Some authors propose the performance of nephrectomy in patients with APKD with pure transperitoneal (TP) or retroperitoneal (RP) laparoscopy. The series of nephrectomies through the TP approach show that, even through they are feasible technically, operative time is very long (more than 4 hours for unilateral nephrectomies) (14-16).
As to the RP approach, the need for an extended previous experience in retroperitonoscopy is described for surgical success. A special disadvantage of this approach is the need to reposition the patient to access the kidney in the opposite side, a procedure that requires around $45 \mathrm{~min}$. and that is not necessary in the cases of TP or HAL approach (17). Recent series using the HAL technique, reporting a shorter operative time, with less morbidity and mortality, can rekindle interest for a more precautious performance of nephrectomies in patients with $\operatorname{APKD}(7,18,19)$.

The performance of a bilateral nephrectomy at once offers some advantages over the two-step procedure, including the need of only one anesthetic and hospital stay, lesser risk of incisional complications and manipulation of previous surgery adherences. It is frequently difficult to define with certainty the side responsible for the origin of the painful symptoms and in this case it is prudent the simultaneous removal of both kidneys for a maximum relief of symptoms (17). In some cases, the performance of a nephrectomy is essential as preparation for a transplant (20). In our 3 patients with APKD, there was a low back pain control without the need of pain medicines beyond the 5 th postoperative day. The complications rate of this series of special patients was not significantly higher than other series of laparoscopic nephrectomy (Table-2) and, especially, operative time for nephrectomy in APKD patients was shorter than the one found in literature. Our case of conversion occurred in a patient with APKD and infected cysts.

\section{CONCLUSIONS}

HAL nephrectomy is an attractive alternative to the treatment of large renal specimens in the presence or not of significant inflammation. In cases that would be difficult to perform exclusively through laparoscopy, HAL allows the dissection to be done in a more agile and safer way, keeping the advantages of a minimally invasive treatment.

\section{CONFLICT OF INTEREST}

None declared. 
Table 2 - Laparoscopic treatment results in many series of nephrectomies for large renal specimens.

\begin{tabular}{|c|c|c|c|c|c|c|c|}
\hline Author & $\begin{array}{c}\text { N of } \\
\text { Kidneys }\end{array}$ & $\begin{array}{c}\text { Operative } \\
\text { Time }\end{array}$ & $\begin{array}{c}\text { Major } \\
\text { Complications }\end{array}$ & $\begin{array}{c}\text { Minor } \\
\text { Complications }\end{array}$ & $\begin{array}{c}\text { Conversion } \\
\text { Rate }\end{array}$ & $\begin{array}{l}\text { Hospital } \\
\text { Stay }\end{array}$ & $\begin{array}{c}\text { Convalescence } \\
\text { Time }\end{array}$ \\
\hline \multicolumn{8}{|c|}{ Laparoscopic Surgery Exclusively Through Transperitoneal Approach to APKD } \\
\hline Elashry, 1996 & 2 & $272 \min *$ & $0 \%$ & $100 \%$ & 0 & 3.5 days & 14 days \\
\hline Dunn, 2000 & 11 & $378 \min *$ & $55 \%$ & $18 \%$ & 0 & 3 days & 35 days \\
\hline Seshadri, 2001 & 10 & $247 \min *$ & 0 & $10 \%$ & $10 \%$ & 2.6 days & Non disclosed \\
\hline
\end{tabular}

Laparoscopic Surgery Exclusively Through Retroperitoneal Approach to APKD

\begin{tabular}{|c|c|c|c|c|c|c|c|}
\hline Gill, 2001 & 20 & $264 \min * *$ & 0 & $50 \%$ & 0 & 1.5 days & 48 days \\
\hline Game, 2003 & 7 & $275 \min *$ & 0 & $28 \%$ & 0 & 11 days & Non disclosed \\
\hline \multicolumn{8}{|c|}{ HAL Surgery to APKD } \\
\hline Rehman, 2001 & 6 & $330 \min * *$ & 0 & 0 & 0 & 4.3 days & 14 days \\
\hline Jenkins, 2002 & 8 & $286 \min * *$ & $25 \%$ & $75 \%$ & 0 & Non disclosed & 14 days \\
\hline Luke, 2004 & 1 & $360 \min *$ & 0 & 0 & 0 & 3 days & Non disclosed \\
\hline
\end{tabular}

Exclusive Laparoscopic Surgery to Giant Hydronephrosis

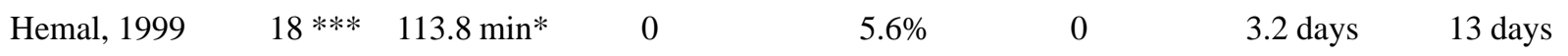

$H A L=$ hand-assisted laparoscopic access; $A P K D=$ adult polycystic kidney disease $; *$ operative times corresponding to unilateral nephrectomy; ** operative times corresponding to bilateral nephrectomies; *** 6 procedures performed through transperitoneal laparoscopic access and 12 procedures through retroperitoneal laparoscopic access.

\section{REFERENCES}

1. Tan YH, Siddiqui K, Preminger GM, Albala DM: Hand-assisted laparoscopic nephrectomy for inflammatory renal conditions. J Endourol. 2004; 18: 770-4.

2. Tobias-Machado M, Lasmar MT, Batista LT, Forseto Jr PH, Juliano RV, Wroclawski ER: Laparoscopic nephrectomy in inflammatory renal disease: proposal for a staged approach. Int Braz J Urol. 2005; 31: 22-8.

3. Nakada SY, Moon TD, Gist M, Mahvi D: Use of the PneumoSleeve as an adjunct in laparoscopic nephrectomy. Urology. 1997; 49: 612-3.

4. Wolf JS Jr, Merion RM, Leichtman AB, Campbell DA Jr, Magee JC, Ounch JD, et al.: Randomized controlled trial of hand-assisted laparoscopic versus open surgical live donor nephrectomy. Transplantation. 2001; 72: 284-90.

5. Stifelman MD, HulL D, Sosa RE, Su LM, Hyman M, Stubenbord W, et al.: Hand assisted laparoscopic donor nephrectomy: a comparison with the open approach. J Urol. 2001; 166: 444-8.
6. Wolf JS Jr, Moon TD, Nakada SY: Hand assisted laparoscopic nephrectomy: comparison to standard laparoscopic nephrectomy. J Urol. 1998; 160: 22-7.

7. Rehman J, Landman J, Andreoni C, McDougall EM, Clayman RV: Laparoscopic bilateral hand assisted nephrectomy for autosomal dominant polycystic kidney disease: initial experience. J Urol. 2001; 166: 427.

8. Stifelman MD, Handler T, Nieder AM, Pizzo JD, Taneja S, Sosa RE, et al.: Hand-assisted laparoscopy for large renal specimens: a multi-institutional study. Urology. 2003; 61: 78-82.

9. Hemal AK, Wadhwa SN, Kumar M, Gupta NP: Transperitoneal and retroperitoneal laparoscopic nephrectomy for giant hydronephrosis. J Urol.1999; 162: 35-9.

10. Gabow PA: Autosomal dominant polycystic kidney disease. N Engl J Med. 1993; 323: 332-42.

11. Brazda E, Ofner D, Riedman B, Spechtenhauser B, Margreiter R: The efect of nephrectomy on the outcome of renal transplantation in patients with poly- 
cystic kidney disease. Ann Transplant. 1996; 1: 1517.

12. Lazarus JM, Bailey GL, Hampers CL, Merrill JP: Hemodialisys and transplantation in adults with polycystic kidney disease. JAMA. 1971; 217: 1821-4.

13. Ho-Hsieh H, Novick AC, Steinmuller D, Streem SB, Buszta C, Goormastic M: Renal transplantation for end-stage polycystic renal disease. Urology. 1987; 30: 322-6.

14. Seshadri PA, Poulin EC, Pace D, Schlachta CM, Cadeddu MO, Mamazza J: Transperitonial laparoscopic nephrectomy for giant polycystic kidneys: a case control-study. Urology. 2001; 58: 23-7.

15. Elashry OM, Nakada SY, Wolf JS Jr, McDougall EM, Clayman RV: Laparoscopy for adult polycystic kidney disease: a promising alternative. Am J Kidney Dis. 1996; 27: 224-33.

16. Dunn MD, Portis AJ, Elbahnasy AM, Shalhav AL, Rothstein M, McDougall EM, et al.: Laparoscopic nephrectomy in patients with end stage renal disease and autosomal dominant polycystic kidney disease. Am J Kidney Dis. 2000; 35: 770-2.

17. Gill IS, Kaouk JH, Hobart MG, Sung GT, Schweizer DK, Braun WE: Laparoscopic bilateral syncronous nephrectomy for autosomal dominant polycystic kidney disease: the initial experience. J Urol. 2001; 165: 1093-8.

18. Luke PP, Spodek J: Hand-assisted laparoscopic resection of the massive autosomal dominant polycystic kidney. Urology. 2004; 63: 369-72.

19. Jenkins MA, Crane JJ, Munch LC: Bilateral hand-assisted laparoscopic nephrectomy for autosomal dominant polycystic kidney disease using a single midline handport incision. Urology. 2002; 59: 32-6.

20. Game X, Vaessen C, Mouzin M, Mallet R, Malavaud B, Sarramon JP, et al.: Retroperitonial laparoscopic nephrectomy for polycystic kidney: preliminary results. Prog Urol. 2003; 13: 215-21.

Received: April 6, 2005 Accepted after revision: August 30, 2005

\section{Correspondence address:}

Dr. Marcos Tobias-Machado

Rua Graúna, 104/131

São Paulo, SP, 04514-000, Brazil

Fax: +55 11 288-1003

E-mail: tobias-machado@uol.com.br 